\title{
Hand-Held Shoulder Strength Measures Correlate With Isokinetic Dynamometry in Elite Water Polo Players
}

\author{
Félix Croteau, Shawn M. Robbins, and David Pearsall
}

\begin{abstract}
Context: Previous authors suggest that lack of strength is an important risk factor for injuries in water polo. Hand-held dynamometers have potential as a clinical tool to measure strength, but they have not been validated in water polo players. Objective: The purpose of this study was to estimate intertrial variability and concurrent validity of hand-held dynamometer shoulder strength measurements in elite water polo players. Methods: A total of 19 male and 20 female elite water polo players performed isometric external (ER) and internal (IR) rotation strength tests against a hand-held dynamometer bilaterally in supine position with the shoulder in a 90-90 position. In addition, concentric IR and ER was captured at $90 \mathrm{deg} / \mathrm{s}$ with an isokinetic dynamometer, and torque values were determined near the 90-90 position. Main Outcome Measures: Spearman correlation coefficients were calculated for ER torque, IR torque, and ER/IR ratios between the devices. Two-way mixed-model intraclass correlations were used to assess intertrial variability. Results: Correlations between the devices were strong to very strong $(\rho=.65-.82, P<.01)$ for absolute IR and ER but low for ER/IR ratios $(\rho=.29, P=.07)$. There was less agreement at higher torque values. Intertrial variability was low with intraclass correlation values .88 to $.93, P<.05$. Conclusions: These results show that hand-held dynamometers are adequate clinical alternatives to measure absolute shoulder strength in water polo players. Stronger players may require stronger evaluators to resist the player's push and obtain reliable results.
\end{abstract}

Keywords: dynamometer, athlete, aquatic

Water polo is the aquatic discipline with the highest rates of injuries (19.4\% injury rates at the 2016 Rio Olympics). ${ }^{1}$ Prospective studies have shown that the shoulder is the most commonly reported site of overuse pain. ${ }^{2}$ Strength factors that relate to shoulder injuries in water polo include strength deficits in internal (IR) or external rotation (ER) as well as low ratios of ER/IR. ${ }^{3}$ The gold standard tools to assess strength are isokinetic dynamometers, but they are costly and time consuming and require trained technicians to operate. Hand-held dynamometers are an alternative tool for shoulder strength IR and ER assessments in healthy adults with good interrater reliability (intraclass correlation $[\mathrm{ICC}]=.89-.97$, SEM $=4.15-$ $13.57 \mathrm{~N}$, and minimal detectable change $=11.28-31.18 \mathrm{~N}$ ), but evaluator strength and experience can potentially induce systematic bias. ${ }^{3,4}$ Thus, the main objective is to assess the intertrial variability of the hand-held dynamometer for measuring shoulder strength and determine its concurrent validity against an isokinetic measurement in elite water polo players. A secondary objective is to compare these findings between males and females.

\section{Methods}

\section{Participants}

Participants were 19 male and 20 female elite water polo players $>18$ years of age (mean 23.1 [3.6] y) and members of the Canadian

Croteau is with the School of Physical and Occupational Therapy, McGill University, Montréal, QC, Canada; and the Institut National du Sport du Québec, Montréal, QC, Canada. Robbins is with the Centre for Interdisciplinary Research in Rehabilitation, Layton-Lethbridge-MacKay Rehabilitation Centre, School of Physical and Occupational Therapy, McGill University, Montréal, QC, Canada. Pearsall is with the Department of Kinesiology and Physical Education, McGill University, Montréal, QC, Canada. Croteau (felix.croteau@ mail.mcgill.ca) is corresponding author. national water polo teams who trained in a full-time program (Table 1). Players with an injury resulting in an inability to fully participate in all regular unmodified training were excluded. All eligible participants at our center were included to maximize sample size. All participants signed a consent form. The study was approved by the McGill University Ethics Institutional Review Board.

\section{Procedures}

All strength measurements were taken in the afternoon before training sessions. Isometric IR and ER strength was measured bilaterally with a hand-held dynamometer (MEDUP ${ }^{\circledR}, 5000 \mathrm{~N}$ full scale and 2000-Hz sampling rate; Atlas Medic, Quebec, Canada) with the patients lying in supine position with the shoulder at $90^{\circ}$ of abduction and ER and the elbow flexed to $90^{\circ}$ (Figure 1). The evaluator (F.C.) was a 95-kg male physiotherapist with 9 years of clinical experience. The evaluator was standing, and the pressure sensor was aligned with the distal radioulnar joint line. Instructions to participants were, "Try to hold this position and keep me from moving you." Three consecutive measurements were taken to resist abduction/ER with a 10-second rest in-between, followed by 3 consecutive measurements of adduction/IR. The participants received verbal encouragement throughout. The sequence was then repeated for the opposite arm.

After a 10-minute break, participants were evaluated in the same position using a CON-TREX ${ }^{\circledR}$ isokinetic dynamometer (CON-TREX MJ; CMV AG, Dübendorf, Switzerland) with a protocol of $90 \mathrm{deg} / \mathrm{s}$ concentric/concentric contractions with a maximum torque tolerance of $250 \mathrm{~N} \cdot \mathrm{m}$ sampled at $4000 \mathrm{~Hz}$ (Figure 2). Eccentric protocols were avoided to minimize impact on training readiness because they induce the most muscle soreness. ${ }^{5} \mathrm{~A}$ warm-up of 5 repetitions was done concentrically for the shoulder internal and external rotators. After a 1-minute break, testing followed for one set of 3 consecutive repetitions. ${ }^{6}$ The 
Table 1 Demographic Characteristics of Participants

\begin{tabular}{|c|c|c|}
\hline Variable & Males $(n=19)$ & Females $(n=20)$ \\
\hline Age, y, mean (SD) & $22(3)$ & $24(4)$ \\
\hline BMI, $\mathrm{kg} / \mathrm{m}^{2}$, mean (SD) & $25.8(2.6)$ & $24.2(2.6)$ \\
\hline \multicolumn{3}{|l|}{ Hand dominance } \\
\hline Right & 19 & 18 \\
\hline Left & 0 & 2 \\
\hline \multicolumn{3}{|l|}{ Player position } \\
\hline Goalie & 3 & 4 \\
\hline Set & 7 & 6 \\
\hline Driver & 9 & 10 \\
\hline \multicolumn{3}{|l|}{ Training setting } \\
\hline National center & 10 & 3 \\
\hline Professional & 8 & 8 \\
\hline College (NCAA) & 1 & 9 \\
\hline
\end{tabular}

Abbreviations: BMI, body mass index; NCAA, National Collegiate Athletic Association.

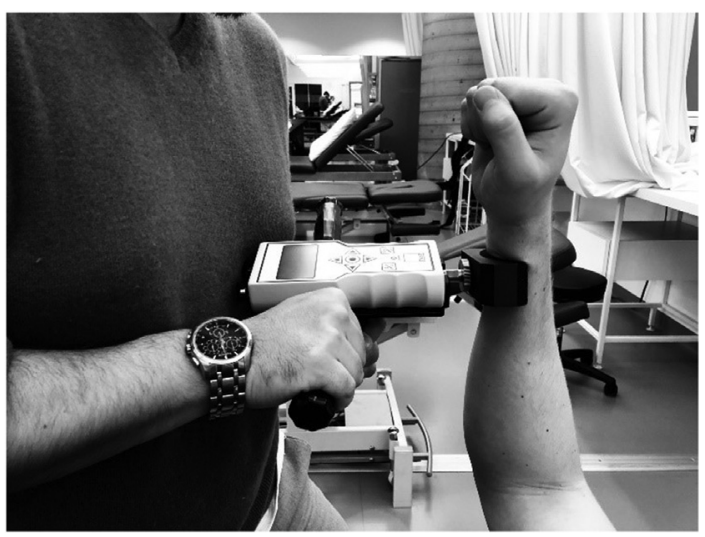

Figure 1 - Experimental setup for hand-held dynamometer. MEDUP ${ }^{\mathrm{TM}}$ hand-held device placed on the ventral aspect of the wrist to test isometric strength in internal rotation with the participant in supine in 90-90 position.

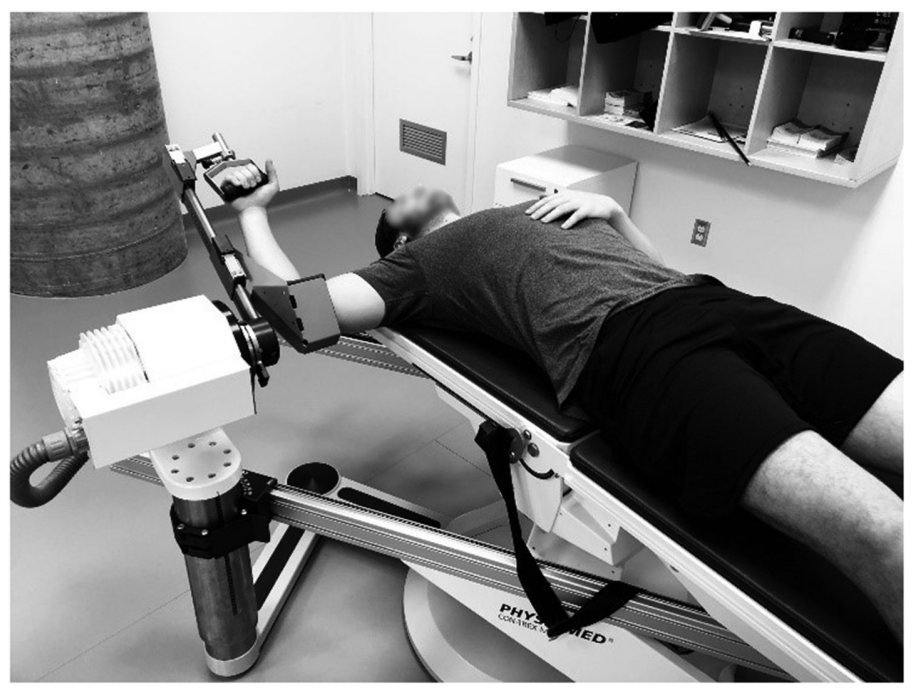

Figure 2 - Experimental setup for isokinetic strength testing. Participant in supine on the Contrex ${ }^{\mathrm{TM}}$ device to measure isokinetic shoulder internal and external rotation in the $90-90$ position. raw signal was gravity corrected automatically as per CON-TREX ${ }^{\circledR}$ software, then a custom RStudio (RStudio Team, PBC, Boston, MA) script filtered the data to maintain only the values measured within the target isokinetic load range at the target of $90(0.5) \mathrm{deg} / \mathrm{s}$. This removed values obtained close to the end of range where the device must slow down, come to a stop, and change direction. Next, the data were filtered to select only the data measured approximately in the same position as the isometric procedure (neutral ER [ $\left.5^{\circ}\right]$ ). This was done to avoid introducing bias from differences in lengthtension relationships obtained in other angles. ${ }^{7}$ The peak value was identified as the maximum value recorded within this filtered subset.

\section{Statistical Analyses}

Mean values of isometric ER and IR strength from the hand-held dynamometer were calculated from the 3 trials. Values were recorded on the device in foot pounds and converted to Newtons. The value was multiplied by the estimated forearm length (in meters) using formulas based on body height from Mohanty et $\mathrm{al}^{8}$ to obtain torque (in kilogram force meter). Isometric ER/IR ratios were calculated by dividing the average external torque by the average internal torque. Peak values of ER and IR concentric strength were obtained by identifying the single maximum overall values in the filtered data from the isokinetic device as per Edouard et al. ${ }^{7}$ Relative strength was obtained by dividing the mean absolute strength of each athlete by their body weight.

Shapiro-Wilk tests confirmed that the data were not normally distributed. Thus, Spearman rank correlation test was used to determine the relationship between isometric and isokinetic strength results using previous criteria (.00-.19 very weak, .20-.39 weak, $.40-.59$ moderate, $.60-.79$ strong, and $.80-1.00$ very strong).$^{9}$ BlandAltman plots were used to measure agreement throughout the range of values obtained, including calculating limits of agreement with 95\% confidence intervals. ${ }^{10}$ Kendall $\tau$ statistic was applied to verify heteroscedasticity (whether the agreement between measures changes as the values increase), using a cutoff value of $\tau>.1{ }^{11}$ Same-day intertrial variability was analyzed using ICC model 3,1 (2way mixed model). ${ }^{4}$ The SEM was calculated by $\mathrm{SD} \times 1 \sqrt{1-\mathrm{ICC}}$. The minimal detectable change was calculated as SEM $\times 1.96 \times \sqrt{2}$. Wilcoxon rank-sum test evaluated differences between sexes. Effect sizes were calculated using Hedge correction $g$ given the small sample size and presented with $95 \%$ confidence intervals. 


\section{Results}

The ER and IR strength values showed strong to very strong9 correlation $(\rho=.65-.82, P<.01)$ between the hand-held and isokinetic dynamometers. However, there was low correlation between ER/IR ratios measured by the 2 devices $(\rho=.26-.29$, $P=.07-.11)$. Bland-Altman plots showed heteroscedasticity for IR and ER: as measurement values increased, the difference between the devices increased as evidenced by the asymmetric funnel shape

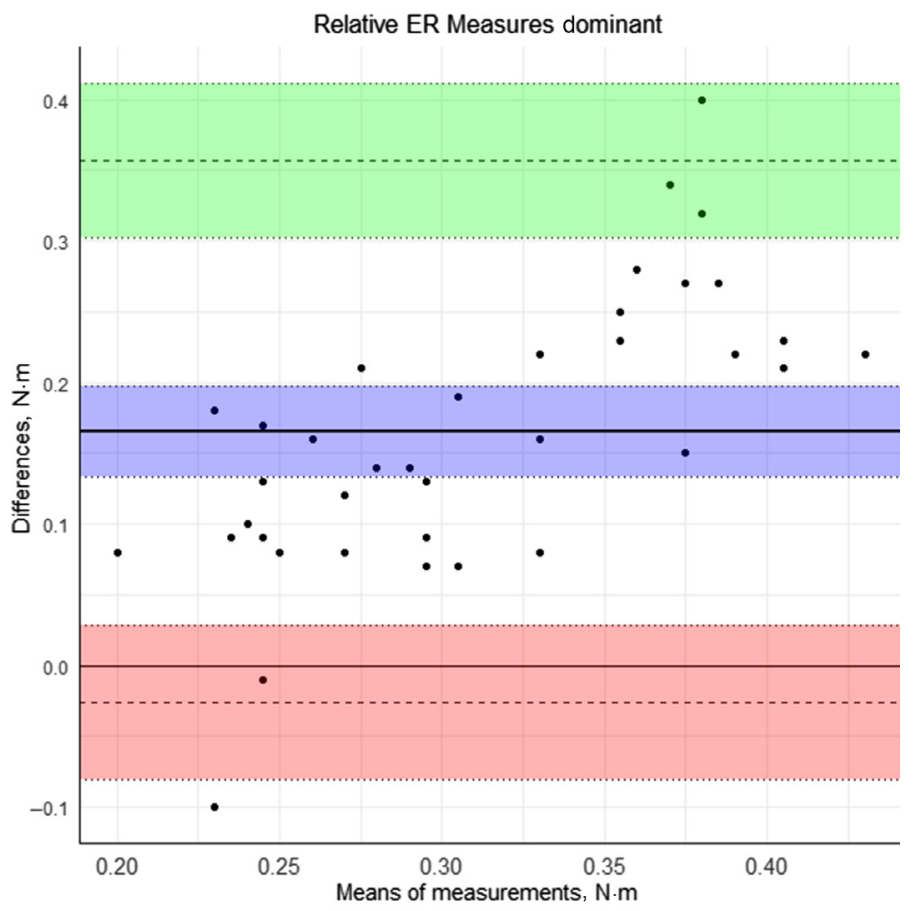

Figure 3 - Bland-Altman plot showing the relationship between the difference versus the mean of measures of shoulder external rotation. The bias line is in the center $(.17,95 \%$ CI .13 to .20$)$, with the upper $(.36,95 \%$ CI .30 to $.41)$ and lower $(-.03,95 \% \mathrm{CI}-.08$ to .03$)$ limits of agreement with their $95 \%$ CIs in green and red, respectively (see Table 2). The shape of the plot shows that as the strength values increase, so does the difference between the two methods. CI indicates confidence interval; ER, external rotation.
(Figure 3 and Supplementary Figure S1 [available online]). Kendall $\tau$ statistic confirmed this trend ( $\tau=.444-.489$ ), and taking the $\log$ values of the variables did not change this trend. Bias values were all positive, indicating that the isokinetic values were higher than matched values on the hand-held device (Table 2). Upper and lower limits of agreement are indicated on the figures with their $95 \%$ confidence intervals. Intertrial variability for the hand-held dynamometer measurements was good to excellent $(\mathrm{ICC}=.88-.93$, $P<.05$; Table 3 ).

Comparisons between male and female participants showed all absolute and relative strength values to be significantly higher for male participants, with moderate to large effect sizes (Supplementary Table S1 [available online]). Strength ratios were not significantly different between sexes.

\section{Discussion}

The hand-held dynamometer in this study showed very good intertrial variability and concurrent validity with the isokinetic dynamometer $(\rho=.65-.82)$ for measuring IR and ER shoulder strength in water polo players, consistent with the general population. ${ }^{4}$ This suggests that hand-held dynamometers can be used to measure shoulder strength in water polo players, although modifications to the protocol may be required for stronger players.

The ICC is a relative reliability measure, and current results show acceptable reliability (>.70) (or low intertrial variability; ICC $=.88-.93$ ). These results are comparable with those obtained by Cools et al, ${ }^{12}$ who conducted a study of 201 overhead throwing athletes with the same testing position ( $\mathrm{ICC}=.86-.92)$. Furthermore, measures of absolute reliability, ${ }^{7}$ such as the SEM, show that the hand-held dynamometer used has an error of 6.6 to $8.3 \mathrm{~kg} \cdot \mathrm{F}$. The minimal detectable change is a measure that suggests that betweentrial differences of at least 8.3 to $10.4 \mathrm{~kg} \cdot \mathrm{F}$ must be measured for the evaluator to conclude that change has occurred. Altogether, these findings show that there is low same-day variability with the handheld device, which makes it an acceptable alternative for testing shoulder strength in water polo players in a clinical setting.

Overall, agreement between hand-held and isokinetic dynamometers was strong, and thus, the hand-held devices can be used clinically when the latter are unavailable. However, ER/IR ratios had low, nonsignificant correlations. This may be the consequence

Table 2 Bias and LoA Between Devices

\begin{tabular}{lcccccc}
\hline Variable (95\% Cl) & Dominant ER & Dominant IR & Nondominant ER & Nondominant IR & Dominant ER/IR & Nondominant ER/IR \\
\hline Bias & $.17(.13$ to .20$)$ & $.23(.20$ to .27$)$ & $.17(.14$ to .20$)$ & $.21(.17$ to .25$)$ & $-.08(-.13$ to -.03$)$ & $-.03(-0.07$ to 0.02$)$ \\
Upper LoA & $.36(.30$ to .41$)$ & $.45(.39$ to .51$)$ & $.35(.30$ to .40$)$ & $.45(.38$ to .52$)$ & $.22(.13$ to .30$)$ & $.25(.17$ to .33$)$ \\
Lower LoA & $-.03(-.08$ to .03$)$ & $.02(-.04$ to .08$)$ & $-.01(-.06$ to .05$)$ & $-.03(-.09$ to .04$)$ & $-.38(-.47$ to -.29$)$ & $-.30(-.38$ to -.22$)$ \\
Kendall $\tau$ & .489 & .444 & .559 & .567 & .175 & .090 \\
\hline
\end{tabular}

Abbreviations: CI, confidence interval; ER, external rotation; IR, internal rotation; LoA, limit of agreement.

Table 3 Intertrial Variability and Concurrent Validity of the Hand-Held Dynamometer

\begin{tabular}{|c|c|c|c|c|c|c|}
\hline Variable $(95 \% \mathrm{Cl})$ & Dominant ER & Dominant IR & Nondominant ER & Nondominant IR & Dominant ER/IR & Nondominant ER/IR \\
\hline $\operatorname{ICC}(3,1)$ & $.90(.84$ to .94$)$ & $.91(.86$ to .95$)$ & $.88(.80$ to .93$)$ & $.93(.89$ to .96$)$ & - & - \\
\hline $\mathrm{SEM}, \mathrm{Kg} \cdot \mathrm{F}$ & $6.6(5.1$ to 8.4$)$ & $8.3(6.2$ to 10.3$)$ & $6.9(5.2$ to 8.9$)$ & $7.7(6.2$ to 10.9$)$ & - & - \\
\hline $\mathrm{MDC}, \mathrm{Kg} \cdot \mathrm{F}$ & $8.3(6.5$ to 10.6$)$ & $10.4(7.8$ to 13.0$)$ & $8.6(6.6$ to 11.1$)$ & $9.7(8.1$ to 13.7$)$ & - & - \\
\hline Spearman $\rho$ & $.65(.42$ to .80$)$ & $.82(.68$ to .90$)$ & .77 (.58 to .87$)$ & $.71(.51$ to .84$)$ & $.29(-.03$ to .56$)$ & $.26(-.06$ to .53$)$ \\
\hline
\end{tabular}

Abbreviations: CI, confidence interval; ER, external rotation; ICC, intraclass correlation; IR, internal rotation; MDC, minimal detectable change. 
of small variability in ER/IR ratios compared with absolute strength measures in this small sample. The ER/IR ratios have shown no correlation with injury in previous studies (Supplementary Figure S2 [available online]). ${ }^{13}$ Bland-Altman plots show increasing disagreement between the 2 testing devices as torque values increase, which is often observed for ratio variables. ${ }^{11}$ This might indicate that hand-held dynamometer strength values are less valid at higher values, and hence, other testing protocols, such as stabilizing the elbow of the participants, must be explored. Otherwise, values recorded with the isokinetic device may be less precise when getting closer to the maximum torque allowed for the test.

Sex comparisons demonstrated that males were significantly stronger than females in both ER and IR muscle groups. However, there were no significant ER/IR ratio differences between sexes. These findings are aligned with other previous studies. ${ }^{3,12}$ Therefore, the authors suggest that careful selection of evaluators be made to assess isometric torque of male water polo players (stronger evaluators when available).

Strength is a characteristic that can change over time, and the ability to measure this more often can have benefits for tracking changes in a sports population. ${ }^{14}$ Although hand-held dynamometers offer a superior clinical usefulness via cheaper costs and less specialized resources, they fail to provide an ongoing measure in the natural sports environment. Wearable technologies, such as inertial measurement units or force gauges, have the potential to provide this type of analysis in real time and on an ongoing basis. ${ }^{15}$ Further developments remain to decrease the error estimates of these technologies for monitoring purposes.

\section{Limitations}

A multievaluator design would have further allowed for interrater analysis, but the main objective was to compare the validity of handheld dynamometers with isokinetic devices in a water polo population. This implies that the findings from this study may only be reproducible for experienced evaluators as well. Conclusions of this study cannot necessarily be generalized to other overhead sports or the general population. Finally, a methodology of multiple speed assessments and both concentric and eccentric measures on the isokinetic device would have yielded further information. One participant with a history of shoulder labrum repair did not feel comfortable with the isokinetic testing and was excluded from the analysis.

\section{Conclusions}

Hand-held dynamometers are a valid, accessible clinical method to assess shoulder ER and IR strength in an elite water polo population. There was strong agreement between the 2 devices for absolute strength. There is less agreement at higher strength values. Therefore, we would recommend maintaining the use of a single device for longitudinal follow-ups in a group of players.

\section{Acknowledgments}

The research team would like to thank the Institut National du Sport du Québec for permission to use their facilities and equipment to collect the data in this study. The Research, Innovation and Dissemination of Information Program funded this study. The authors have no conflict of interest to declare.

\section{References}

1. Mountjoy M, Miller J, Junge A. Analysis of water polo injuries during 8904 player matches at FINA World Championships and Olympic games to make the sport safer. Br J Sports Med. 2019;53(1):25-31. PubMed ID: 30194222 doi:10.1136/bjsports-2018-099349

2. Hams A, Evans K, Adams R, Waddington G, Witchalls J. Epidemiology of shoulder injury in sub-elite level water polo players. Phys Ther Sport. 2019;35:127-132. PubMed ID: 30551122 doi:10.1016/j. ptsp.2018.12.001

3. Hams AH, Evans K, Adams R, Waddington G, Witchalls J. Shoulder internal and external rotation strength and prediction of subsequent injury in water-polo players. Scand J Med Sci Sports. 2019;29(9): 1414-1420. PubMed ID: 31066126 doi:10.1111/sms.13459

4. Holt KL, Raper DP, Boettcher CE, Waddington GS, Drew MK. Hand-held dynamometry strength measures for internal and external rotation demonstrate superior reliability, lower minimal detectable change and higher correlation to isokinetic dynamometry than externally-fixed dynamometry of the shoulder. Phys Ther Sport. 2016; 21:75-81. PubMed ID: 27500450 doi:10.1016/j.ptsp.2016.07.001

5. Stark T, Walker B, Phillips JK, Fejer R, Beck R. Hand-held dynamometry correlation with the gold standard isokinetic dynamometry: a systematic review. $P M \& R$. 2011;3(5):472-479. PubMed ID: 21570036 doi:10.1016/j.pmrj.2010.10.025

6. Papotto BM, Rice T, Malone T, Butterfield T, Uhl TL. Reliability of isometric and eccentric isokinetic shoulder external rotation. J Sport Rehabil. 2016;25(2):01. doi:10.1123/jsr.2015-0046

7. Edouard P, Samozino P, Julia M, et al. Reliability of isokinetic assessment of shoulder-rotator strength: a systematic review of the effect of position. 2011;20(3):367. PubMed ID: 21828388 doi:10.1123/jsr.20.3.367

8. Mohanty BB, Agrawal D, Mishra K, Samantsinghar P, Chinara PK. Estimation of height of an individual from forearm length on the population of Eastern India. J Med Alli Sci. 2013;3(2):72-75.

9. Evans JD, Wuensch KL. Straightforward statistics for the behavioral sciences. J Am Stat Assoc. 1996;91(436):1750.

10. Bland JM, Altman DG. Agreement between methods of measurement with multiple observations per individual. $J$ Biopharm Stat. 2007;17(4):571-582. doi:10.1080/10543400701329422

11. Brehm MA, Scholtes VA, Dallmeijer AJ, Twisk JW, Harlaar J. The importance of addressing heteroscedasticity in the reliability analysis of ratio-scaled variables: an example based on walking energy-cost measurements. Develop Med Child Neurol. 2012;54(3):267-273. PubMed ID: 22150364 doi:10.1111/j.1469-8749.2011.04164.x

12. Cools AMJ, Vanderstukken F, Vereecken F, et al. Eccentric and isometric shoulder rotator cuff strength testing using a hand-held dynamometer: reference values for overhead athletes. Knee Surg Sports Traumatol Arthrosc. 2016;24(12):3838-3847. PubMed ID: 26294055 doi:10.1007/s00167-015-3755-9

13. Hams A, Evans K, Adams R, Waddington G, Witchalls J. Reduced shoulder strength and change in range of motion are risk factors for shoulder injury in water polo players. Phys Ther Sport. 2019;40:231237. PubMed ID: 31629168 doi:10.1016/j.ptsp.2019.10.003

14. Bittencourt NFN, Meeuwisse WH, Mendonça LD, Nettel-Aguirre A, Ocarino JM, Fonseca ST. Complex systems approach for sports injuries: moving from risk factor identification to injury pattern recognition-narrative review and new concept. $\mathrm{Br} J$ Sports Med. 2016;50(21):1309-1314. doi:10.1136/bjsports-2015-095850

15. Picerno P, Viero V, Donati M, Triossi T, Tancredi V, Melchiorri G. Ambulatory assessment of shoulder abduction strength curve using a single wearable inertial sensor. J Rehabil Res Develop. 2015;52(2): 171-180. PubMed ID: 26230401 doi:10.1682/JRRD.2014.06.0146 


\section{Erratum: Croteau et al. (2021)}

In the original publication of this article, Kendall $\tau$ numbers were inaccurately written in the first paragraph of the Results section. In addition, statistics were inaccurately written in Table 2, Figure 3, and the Supplemental Material. The online version of this article has been corrected. The authors apologize for this error 